\title{
Dual DTM-Padé approximations on free convection MHD mass transfer flow of nanofluid through a stretching sheet in presence of Soret and Dufour Phenomena
}

\author{
DIBYENDU SAHA and SANJIB SENGUPTA \\ Department of Mathematics \\ Assam University \\ Silchar-788011, Assam \\ INDIA
}

\begin{abstract}
A theoretical study is made to investigate heat and mass transfer analysis on the single phase flow of an electrically conducting, Al2O3-Water nanofluid over a linearly stretching sheet in presence of Soret and Dufour effects. An applied magnetic field is considered normal to the flow, while the effect of induced magnetic field got neglected for small magnetic Reynolds number's value of the flow field relative to the applied field. Since voltage difference at the lateral ends of the sheet is very small, the influence of the electric field is thus omitted. The governing equations representing the physical model of the fluid flow is solved by means of DTM-Padé approximations. The acquired results show that an increase in the Soret number (Dufour number) decreases (increases) the temperature profiles but increases (decreases) the concentration profiles. The axial velocity profiles found decreasing with increasing values of the magnetic parameter. Both chemical reaction and thermal radiation parameters maximize the temperature profiles whereas a reverse phenomenon is seen on concentration profiles. The obtained tables show that increasing nanoparticle volume fraction escalates skin-friction coefficient, Nusselt number and Sherwood number whereas an increase in Richardson number decreases the Nusselt number but increases the Sherwood number.
\end{abstract}

Key-Words: Nanofluid, Magnetohydrodynamics, Soret and Dufour effects, DTM-Padé approximations, Thermal radiation, Chemical reaction

Received: September 9, 2019. Revised: February 11, 2020. Accepted: February 18, 2020. Published: February 24, 2020.

\section{Introduction and background}

There are a variety of industries like plastic manufacturing, automotive, electronic, transportation, power, aerospace industries, where the demand for heat exchanger devices is increasing rapidly. To fit the purpose, the devices are to be lighter in weight and smaller in size. Also, they must provide higher performance in terms of heat transfer. Conventional fluids that are being used for heat transfer such as water oil, engine oil, and ethylene glycol have a limited thermal conductivity of heat transfer, resulting in a limitation for enhancing the performance of most of the electronic devices used for the engineering applications. To enhance the thermal conductivity of these fluids, a considerable amount of researches have been made. It was Choi [1], who first invented a new type of fluid by suspending small volumetric quantities of nanometer- sized (diameters less than 50nm) particles, called nanoparticles, in the base fluid and termed it as nanofluid. These new type of fluid was developed by considering nanoparticles like $\mathrm{Cu}, \mathrm{Ag}$, $\mathrm{CuO}, \mathrm{TiO}_{2}, \mathrm{Al}_{2} \mathrm{O}_{3}, \mathrm{SiO}_{2}$ etc. suspended in a base fluid like water. These nanofluids show a considerable heat transfer enhancement (15\% $40 \%$ ) when compared to conventional fluids. Keblinski et al. [2] have advanced the study on nanofluids and interpreted that the increase in thermal conductivity of nanofluids is due to the effect of nanoparticle clustering, the heat transport in the nanoparticles, and also for Brownian motion of particles. Buongiorno [3] have investigated convective heat transfer of nanofluid in detail. Very recently Sengupta and Karmakar [4] analyzed heat and mass transfer properties on the mixed convective flow of radiative fluid under influence of 
various significant effects and outlined numerous noteworthy findings.

Magnetohydrodynamics (MHD) is the study of the flow of electrically conducting fluids under the influence of magnetic fluid domain. The study of MHD flow of nanofluid over a continuous stretching sheet remains as a streamline research topic since few decades due to its various applications in physics, chemistry, and in industrial processes such as cooling of nuclear reactor, metal casting, polymer technology, petroleum industries, power generators, and magnetic resonance imaging (MRI) etc. El-Kabeir [5] in addition to Haile and Shankar [6] have categorically shown that the rate of cooling can be controlled by using electrically conducting fluid in the presence of the magnetic field. Sengupta [7] described various aspects of MHD flow past a vertical flat surface embedded in Darcian porous media and studied heat and mass transfer phenomena thereof. Many researchers have investigated the effects of different important parameters on various profiles of magnetohydrodynamic nanofluids in different geometries [8, 9, 10, 11]. Sheikholeslami et al. [12] have explored the effect of various parameters on magnetohydrodynamics nanofluid by considering a two-phase fluid flow model.

MHD effect is considered in the boundary layer flow of nanofluid over a stretching sheet, which is a significant area of study among researchers as it has a tremendous applications in many engineering and industrial processes such as manufacturing of rubber and plastic sheets, annealing of copper wires, production of glass fiber, cooling of fiber spinning, irrigation systems, blood vessels etc. In production of rubber and plastic sheets, the die includes a manifold that stretches the polymer melt laterally before it flows through the slit and to achieve high production rates an efficient method of cooling must be integrated with the extrusion process, which is usually done by directing the extrudate into a quenching bath of water or onto chill rolls. Thus stretching of polymer melt brings an orientation in it and the quality of the final product greatly depends on the heat and mass transfer process. Reddy and Chamkha [13] have studied various parametric effects over nanofluid flow past a stretching sheet. Rashidi et al. [14] have investigated the buoyancy effect on the MHD flow of nanofluid over a stretching sheet. Similarity solution to the threedimensional boundary layer flow of a second-grade nanofluid past a stretching surface is explored by
Hayat et al. [15]. A study of the effects of thermodiffusion and thermal radiation on Williamson nanofluid over a porous stretching sheet was proposed by Bhatti and Rashidi [16]. Saha and Sengupta [17] have studied heat transfer analysis on $\mathrm{TiO}_{2}$-water nanofluid past a stretching sheet under influence of magnetic field domain and bordered remarkable conclusions.

Thermal radiation on heat and mass transfer problems of MHD nanofluid flow is gaining much more interest among researchers due to its wide industrial applications. Thermal radiation of heat transfer flow becomes very important in designing spaceships, nuclear power plants, aircraft, wind turbines, solar power, underground nuclear waste disposal, and many more. All most all of these engineering processes take place at a very high temperature and for that, it is essential to consider the radiative heat transfer. Thermal radiation in nanofluids becomes more significant as radiation emission mostly depends on the temperature of the nanoparticles. Also, the thermal radiation effect should be given proper importance when the difference between the ambient temperature and surface temperature is significant and high order of magnitude. Many researchers have contributed towards the study of heat and mass transfer of nanofluids considering thermal radiation effect. Hayat et al. [18] explored MHD three dimensional flow of nanofluid with velocity slip and non-linear thermal radiation and concluded that the heat transfer rate increases when temperature and radiation parameters are increased. Karthikeyan et al. [19] have also shown, by their study on the MHD flow, that temperature increases for increasing values of thermal radiation.

The heat and mass transfer problems of MHD flow of nanofluid with chemical reaction have gained a considerable amount of attention among the researchers due to its rich applications in many industrial processes such as spray drying of milk, energy transfer in a wet cooling tower, evaporation processes etc. Also, it is observed thatgenerally, an increase in volume fraction of nanoparticle increases the reaction rate, as higher volume fraction means that more particles are closer together, so they undergo more collision and have a better chance of reacting, which may influence the flow properties. Chemical reactions are either homogeneous or heterogeneous processes. Reactions which occur uniformly through a given phase are called homogeneous reactions, while those reactions which 
occur within a restricted phase are known as heterogeneous reactions. It is relevant to mention exothermic reactions and endothermic reactions here; the former is a type of chemical reaction which releases heat and provides net energy to its surroundings whereas the latter is opposite in nature to the former and it describes a chemical reaction in which system absorbs energy in terms of heat from its surroundings. Different effects on heat and mass transfer due to a stretching cylinder in saturated porous medium with chemically reactive species were studied by El-Kabeir [5]. Heat and mass transfer in the MHD flow of nanofluids through a porous media due to a permeable stretching sheet with viscous dissipation and chemical reaction effects was investigated by Yirga and Tesfay [11]. Sengupta [20] has considered first order chemical reaction together with radiation absorption effect on two-dimensional free convective flow and has studied the effect of chemical reaction on fluid velocity and concentration profiles. Karthikeyan et al. [19] explored the effects of different significant parameters on magnetohydrodynamic mixed convection heat and mass transfer stagnation point flow towards a vertical plate in a porous medium with chemical reaction.

The thermo-diffusion (Soret) and diffusionthermo (Dufour) effects play an important role in heat and mass transfer processes of MHD flow, when considerable density difference occurs due to large temperature gradients, in the fluid flow regime. Soret and Dufour effects on MHD nanofluids arise in different physical situations like chemical engineering, solar collector, geothermal processes etc. The Soret effect corresponds to the mass flux produced by a temperature gradient and the Dufour effect corresponds to the heat flux produced by a temperature gradient. A good amount of work has been done by the researchers to study the importance of these two effects in fluids with medium and small molecular weights. Ahmed et al. [21] have studied the influence of Soret effect on unsteady MHD flow past a vertical oscillating plate and concluded that velocity and concentration of fluids with medium molecular weights are appreciably influenced by Soret number. Pal et al. [22] studied effects of Soret and Dufour numbers on MHD convective-radiative heat and mass transfer of nanofluids past a vertical stretching/shrinking sheet and also have shown applicable effects of these numbers. Hayat et al. [9] have investigated Soret and Dufour effects in MHD peristalsis of pseudoplastic nanofluid and have found temperature development towards increasing values of Dufour number. Reddy and Chamkha [13] have studied effects of Soret and Dufour number on the MHD convective flow of nanofluids namely $\mathrm{Al}_{2} \mathrm{O}_{3}$-water and $\mathrm{TiO}_{2}$-water past a stretching sheet and have investigated the effects of these numbers on various aspects. Sengupta and Ahmed [23] have discovered effects of Soret and Dufour numbers on MHD free convective mass transfer flow of radiative fluid through a wavy permeable channel.Again, it is well known that modeling of most of the different physical situations that arise in the real world, specifically the one of heat and mass transfer phenomenon of MHD nanofluid flow under various effects, involves highly non-linear differential equations One of the very effective methods to solve these non-linear differential equations is differential transform method (DTM), which was first introduced by Zhou [24], to solve both linear and non-linear initial value problems in the application of electric circuit theory. DTM is a powerful semianalytical technique, which is independent of any small or large quantities, i.e. it can deal with the large quantities that are possibly involved with the highly non-linear differential terms. DTM is based on Taylor series and gives an analytic Taylor series solution for differential equations, also the solution is polynomial in form but when compared to Taylor series method, which is computationally intensive for higher orders, DTM has no such complications to deal with the higher order terms. Heat transfer problems of nanofluids in different geometry are solved by means of DTM by many researchers [25, $26,27,28,16]$. The limitation of DTM is when; it is applied to the differential equations with semi-open boundary conditions, the theory of DTM does not satisfy it. That is, when one of the limiting cases took a sufficiently large value, the power series as obtained by DTM, diverge. Therefore, to deal with the unbounded boundary domain problems, the series solution as obtained by DTM must be combined with the Padé-approximation, so that the solution of DTM can be transferred to a rational function of sufficient finite order, which will then agree with the conditions defined at the unbounded boundary. Researchers [25, 28, 16] have solved MHD boundary layer equations by using DTMPadé technique.

From the ongoing discussions, it is observed that no striking effort has been made so far to study the heat and mass transfer analysis of electrically 
conducting nanofluid over a vertical stretching sheet in presence of physical phenomena like chemical reaction, heat source (generation), Soret, Dufour and thermal radiation in a semi-open boundary set-up by applying the dual applications of DTM-Padé approximations. In this context the present study offers significant analysis of heat and mass transfer phenomenon of $\mathrm{Al}_{2} \mathrm{O}_{3}$-water nanofluid over a linearly stretching sheet in company of Soret and Dufour effects, together with an applied magnetic field, acting normal to the flow. Effects of volume fraction, Richardson number, Magnetic field parameter, thermal radiation parameter and chemical reaction parameter on the Nusselt number, Skin-friction coefficient, and Sherwood number are also studied. The present study may find its possible applications in the field of rubber and dye industries and also in the field of geoengineering.

\section{Formulation of the problem}

The present study deals with a steady, twodimensional, viscous, incompressible, and electrically conducting hydromagnetic boundary layer flow of nanofluids over a vertical stretching sheet, stretched linearly along its length. The $\mathrm{x}$-axis is engaged along the length of the plate in the upward vertical direction whereas the $y$-axis is considered normal to the plate and the flow domain lies in the region $y \geq 0$. A transverse magnetic field of uniform strength has been applied normal to the flow direction, while the effect of the induced magnetic field got neglected due to small magnetic Reynolds number's value of the flow field. Due to the negligible voltage difference, the electrical field is also discarded for the study. The base fluid is taken as water and it is supposed that the base fluid and the solid nanoparticles remain in thermal equilibrium so that no slip occurs between them. Also, heat source phenomenon is being generated within the flow domain. Considering the usual Boussinesq and boundary layer approximations, the governing equations for continuity, momentum, and energy in two-dimensional nanofluid flow model are thus respectively developed as:

$\frac{\partial u}{\partial x}+\frac{\partial v}{\partial y}=0$

$u \frac{\partial u}{\partial x}+v \frac{\partial u}{\partial y}=v_{n f} \frac{\partial^{2} u}{\partial y^{2}}-\frac{\sigma_{n f} B_{0}^{2}}{\rho_{n f}} u-\frac{v_{n f}}{k} u+$
$\frac{g}{\rho_{n f}}\left\{\left(\rho \beta_{T}\right)_{n f}\left(T-T_{\infty}\right)+\left(\rho \beta_{C}\right)_{n f}\left(C-C_{\infty}\right)\right\}$ $u \frac{\partial T}{\partial x}+v \frac{\partial T}{\partial y}=\frac{k_{n f}}{\left(\rho c_{p}\right)_{n f}} \frac{\partial^{2} T}{\partial y^{2}}-\frac{1}{\left(\rho c_{p}\right)_{n f}} \frac{\partial q_{r}}{\partial y}+$

$\frac{1}{\left(\rho c_{p}\right)_{n f}} \frac{D_{M} K_{T}}{c_{S} c_{p}} \frac{\partial^{2} C}{\partial y^{2}}+\frac{Q}{\left(\rho c_{p}\right)_{n f}}\left(T-T_{\infty}\right)$

$u \frac{\partial C}{\partial x}+v \frac{\partial C}{\partial y}=D_{M} \frac{\partial^{2} C}{\partial y^{2}}+\frac{D_{M} K_{T}}{T_{M}} \frac{\partial^{2} T}{\partial y^{2}}-k_{l}\left(C-C_{\infty}\right)$

Subject to the stretching sheet boundary conditions as:

$u=u_{M}(x)=b x, \quad v=v_{M}, \quad T=T_{M}(x)=T_{\infty}+$

$\left(T_{M}-T_{\infty}\right) x, \quad C=C_{M}(x)=C_{\infty}+\left(C_{M}-\right.$

$\left.C_{\infty}\right) x$ at $y=0$

$u \rightarrow 0, T=T_{\infty}, C=C_{\infty}$ at $y \rightarrow \infty$

Where, $T_{M}>T_{\infty}, C_{M}>C_{\infty}$

$\alpha_{n f}=\frac{k_{n f}}{\left(\rho c_{p}\right)_{n f}}$

$\mu_{n f}=\frac{\mu_{f}}{(1-\emptyset)^{2.5}} \mu_{n f}=\frac{\mu_{f}}{(1-\emptyset)^{2.5}}$

$\rho_{n f}=(1-\emptyset) \rho_{f}+\emptyset \rho_{s}$

$\left(\rho c_{p}\right)_{n f}=(1-\emptyset)\left(\rho c_{p}\right)_{f}+\emptyset\left(\rho c_{p}\right)_{s}$

$(\rho \beta)_{n f}=(1-\emptyset)(\rho \beta)_{f}+\emptyset(\rho \beta)_{s}$

$\frac{k_{n f}}{k_{f}}=\frac{\left(k_{s}+2 k_{f}\right)-2 \phi\left(k_{f}-k_{s}\right)}{\left(k_{s}+2 k_{f}\right)+2 \phi\left(k_{f}-k_{s}\right)}$

Following the Rosseland approximation [29], which is applicable to optically thick media, the net radiation heat flux $q_{r}$ is expressed as:

$q_{r}=-\frac{4}{3 A_{R}} \operatorname{grad}\left(E_{b}\right)$

Where $E_{b}$ is given by absolute temperature $T$ by the constant $\sigma_{S B}^{*}$. Also, this is to be assume that due to the radiation within the flow, $T^{4}$ can be expressed as Taylor series about $T_{\infty}$ and can be approximated [22]as:

$T^{4} \approx 4 T_{\infty}^{3} T-3 T_{\infty}^{4}$

Using (9) in (8), we have:

$\frac{\partial q_{r}}{\partial y}=-\frac{16 \sigma_{S B}^{*} T_{\infty}^{3}}{3 A_{R}} \frac{\partial^{2} T}{\partial y^{2}}$

We now introduce the following set of similarity solutions of the Equations(1)-(4) and with boundary conditions (5) and (6) in the following form: 
$\psi(x, y)=\left(b v_{f}\right)^{1 / 2} x f(\eta), \eta=\left(\frac{b}{v_{f}}\right)^{\frac{1}{2}} y, \theta(\eta)=$ $\frac{T-T_{\infty}}{T_{M}-T_{\infty}}, \emptyset^{*}(\eta)=\frac{C-C_{\infty}}{C_{M}-C_{\infty}}$

Such that $u=\frac{\partial \psi}{\partial y}, v=-\frac{\partial \psi}{\partial x}$ which satisfy the equation of continuity (1).

By means of Equations (7) - (11), the Equations(2)-(4) are transformed into the following non-linear ordinary differential equations:

$\frac{1}{\phi_{1} \phi_{2}} f^{\prime \prime \prime}+f f^{\prime \prime}-\left(f^{\prime}\right)^{2}-\frac{1}{\phi_{2}}\left(M^{2}+\frac{k_{p}}{\phi_{1}}\right) f^{I}+$

$\frac{\phi_{4}}{\phi_{2}} \operatorname{Ri}\left(\theta+\xi \emptyset^{*}\right)=0$

$\frac{1}{P r}\left(\frac{k_{n f}}{k_{f}}+N r\right) \theta^{I I}+\phi_{3} f \theta^{I}+Q s \theta+D u \emptyset^{* I I}=0$

$\frac{1}{S c} \emptyset^{* I}+f \emptyset^{*^{I}}+\operatorname{Sr} \theta^{I I}-\operatorname{Cr} \emptyset^{*}=0$

With the following set of boundary conditions as obtained from Equations (5)-(6):

$f(\eta)=S, f^{\prime}(\eta)=1, \theta(\eta)=1, \emptyset^{*}(\eta)=1$ at $\eta=0$

$f^{\prime}(\eta) \rightarrow 0, \theta(\eta) \rightarrow 0, \emptyset^{*}(\eta) \rightarrow 0$ as $\eta \rightarrow \infty$

Equations (12)-(16) are non-dimensional in nature with the following quantities:

$M=\sqrt{\frac{\sigma_{n f^{B_{0}^{2}}}}{b \rho_{f}}}, k_{p}=\frac{\mu_{f}}{\rho_{f}, b . k}, R i=\frac{g\left(\rho \beta_{T}\right)_{f}\left(T_{M}-T_{\infty 0}\right)}{b^{2} x v_{f}}$,

$S c=\frac{v_{f}}{D_{M}}, \xi=\frac{\phi_{5} \beta_{c}\left(C_{M}-C_{\infty}\right)}{\phi_{4} \beta_{T}\left(T_{M}-T_{\infty}\right)}, S r=\frac{D_{M} k_{T}}{v_{f} T_{M}} \frac{T_{M}-T_{\infty}}{C_{M}-C_{\infty}}$,

$C r=\frac{k_{l}}{b}, \operatorname{Pr}=\frac{v_{f}}{\alpha_{f}}, N r=\frac{16 \sigma_{S B}^{*} T_{\infty}^{3}}{3 k_{f} A_{R}}, Q_{s}=\frac{Q}{b\left(\rho c_{p}\right) f}$,

$D u=\frac{D_{M} k_{T}}{v_{f}\left(\rho c_{p}\right)_{f} C_{s} C_{p}} \frac{C_{M}-C_{\infty}}{T_{M}-T_{\infty}}$

Beside the aforesaid terms, we also have:

$\phi_{1}=(1-\phi)^{2.5}, \phi_{2}=1-\phi+\phi\left(\frac{\rho_{s}}{\rho_{f}}\right), \phi_{3}=1-$
$\phi+\phi \frac{\left(\rho c_{p}\right)_{s}}{\left(\rho c_{p}\right)_{f}}, \phi_{4}=1-\phi+\phi \frac{\left(\rho \beta_{T}\right)_{s}}{\left(\rho \beta_{T}\right)_{f}}, \phi_{5}=1-$
$\phi+\phi \frac{\left(\rho \beta_{c}\right)_{s}}{\left(\rho \beta_{c}\right)_{f}}$
The important physical quantities of engineering interest like Nusselt number, Sherwood number, and the skin-friction coefficient, are respectively defined as:

$$
\begin{aligned}
& R e_{x}^{-\frac{1}{2}} N u_{x}=-\frac{k_{n f}}{k_{f}}(1+N r) \theta^{\prime}(0), R e_{x}^{-\frac{1}{2}} S h_{x}= \\
& -\emptyset^{* I}(0), \operatorname{Re}_{x}^{\frac{1}{2}} C_{f}=-\frac{2}{(1-\phi)^{2.5}} f^{\prime \prime}(0)
\end{aligned}
$$

\section{Solution Methodology}

We have used DTM-Padé approximation technique to solve the complex system of coupled non-linear equations. Thus for a better understanding, both DTM method and Padé technique are described separately.

\subsection{The basic idea of DTM}

Consider $f(x)$ to be an analytic function and $x=x_{0}$ be any arbitrary point in the domain. The differential transform of $f(x)$ is represented as the $k^{\text {th }}$ derivative in the following form:

$F[\mathrm{k}]=\frac{1}{k !}\left(\frac{d^{k} f(x)}{d x^{k}}\right)_{x=x_{0}}$

Where $F[k]$ is the transformed function of the original function $f(x)$ under DTM. The inverse transformation is represented as a power series with $x=x_{0}$ as its centre and is defined as follows:

$f(x)=\sum_{k=0}^{\infty}\left(x-x_{0}\right)^{k} F(k)$

Combining Equation (20) and (21), we have

$f(x)=\sum_{k=0}^{\infty} \frac{\left(x-x_{0}\right)^{k}}{k !}\left(\frac{d^{k} f(x)}{d x^{k}}\right)_{x=x_{0}}$

In the expression (22), the form of $f(x)$ is considered up to a suitable number of finite terms, consistent with the convergence of the series and thus Equation (21) can be represented in the following form as:

$f(x)=\sum_{k=0}^{L}\left(x-x_{0}\right)^{k} F[k], L(>0) \in \mathbb{Z}$

Here it is considered that the combination of the terms $\sum_{k=L+1}^{\infty}\left(x-x_{0}\right)^{k} F[k]$ is negligibly small for 
the construction of the convergence of the series solution.

\subsection{The basic idea of Padé approximant}

Consider a function $f(\eta)$ in a power series form as:

$f(\eta)=\sum_{i=0}^{\infty} \alpha_{i} \eta^{i}$

Where $\alpha_{i}$ 's are constants. The Padé Approximant of Equation (20) is a rational function and is represented as:

$$
[L, M]=\frac{a_{0}+a_{1} \eta+a_{2} \eta^{2}+\cdots+a_{L} \eta^{L}}{b_{0}+b_{1} \eta+b_{2} \eta^{2}+\cdots+b_{M} \eta^{M}}
$$

Equation (25) is a Maclaurin's series expansion and will agree with (24) as far as possible. By considering the normalization condition $b_{0}=1$, the total number of independent terms in the numerator and denominator coefficients will be $L+1$ and $M$ respectively. Normally $[L, M]$ ought to fit the Equation (24) through orders $1, \eta, \eta^{2}, \eta^{3}, \ldots, \eta^{L+M}$. The $[L, M]$ approximant can be uniquely determined.

Therefore, we have:

$f(\eta)=\sum_{i=0}^{\infty} \alpha_{i} \eta^{i}=\frac{a_{0}+a_{1} \eta+a_{2} \eta^{2}+\cdots+a_{L} \eta^{L}}{b_{0}+b_{1} \eta+b_{2} \eta^{2}+\cdots+b_{M} \eta^{M}}+$
$O\left(\eta^{L+M+1}\right)$

Which can be further expressed as:

$\left(\alpha_{0}+\alpha_{1} \eta+\alpha_{2} \eta^{2}+\alpha_{3} \eta^{3}+\cdots\right) *\left(1+b_{1} \eta+\right.$ $\left.b_{2} \eta^{2}+\cdots+b_{M} \eta^{M}\right)=\left(a_{0}+a_{1} \eta+a_{2} \eta^{2}+\cdots+\right.$ $\left.a_{M} \eta^{M}\right)+O\left(\eta^{L+M+1}\right)$

Comparing the coefficients of $1, \eta, \eta^{2}, \eta^{3}, \ldots, \eta^{L}$ in Equation (27), we thus obtain the following set of relations between the coefficients:

$a_{0}=\alpha_{0}, a_{1}=\alpha_{1}+\alpha_{0} b_{1}, a_{2}=\alpha_{2}+\alpha_{1} b_{1}+$ $\alpha_{0} b_{2}, \ldots, a_{L}=\alpha_{L}+\alpha_{L-1} b_{1}+\cdots+\alpha_{0} b_{L}$

Again, on comparing the coefficients of $\eta^{L+1}, \eta^{L+2}, \eta^{L+3}, \ldots, \eta^{L+M}$ in Equation (27), we also obtain the following set of linear system of equations as:

$\alpha_{L+1}+\alpha_{L} b_{1}+\cdots+\alpha_{L-M+1} b_{M}=0, \alpha_{L+2}+$

$\alpha_{L+1} b_{1}+\cdots+\alpha_{L-M+2} b_{M}=0$

proceeding in the same way we arrive at $\alpha_{L+M}+\alpha_{L+M-1} b_{1}+\cdots+\alpha_{L} b_{M}=0$
The system of equations (29) consists of $M$ linear equations with $M$ unknown denominators and thus can be expanded in the matrix equation form as:

$$
\begin{aligned}
& \left(\begin{array}{cccc}
\alpha_{L-M+1} & \alpha_{L-M+2} & \cdots & \alpha_{L} \\
\alpha_{L-M+2} & \alpha_{L-M+3} & \cdots & \alpha_{L+1} \\
\vdots & \vdots & \ddots & \vdots \\
\alpha_{L} & \alpha_{L+1} & \cdots & \alpha_{L+M-1}
\end{array}\right) *\left(\begin{array}{c}
b_{M} \\
b_{M-1} \\
b_{M-2} \\
\vdots \\
b_{1}
\end{array}\right)= \\
& -\left(\begin{array}{c}
\alpha_{L+1} \\
\alpha_{L+2} \\
\alpha_{L+3} \\
\vdots \\
\alpha_{L+M}
\end{array}\right)
\end{aligned}
$$

From Equation (30), denominator coefficients $b_{i}$ 's can be found while the numerator coefficients can be calculated from Equation (28). Thus using Equation (26), the $[L, M]$ Padé approximant can be constructed. It is to be noted here that with increasing order of Padé approximant, the accuracy of the solution, increases.

\section{Solution Analysis by DTM-Padé technique}

Using differential transform operator, the Equations (12)-(14) are respectively transformed into an equivalent algebraic set of equations as:

$$
\begin{aligned}
& F[\mathrm{k}+3]=\frac{\phi_{1} \phi_{2}}{(k+1)(k+2)(k+3)}\left[\sum_{r=0}^{k}(r+1)(k-r+\right. \\
& \text { 1) } F[\mathrm{r}+1] F[\mathrm{k}-\mathrm{r}+1]+\frac{1}{\phi_{2}}\left(M^{2}+\frac{k_{p}}{\phi_{1}}\right)(k+ \\
& \text { 1) } F[\mathrm{k}+1]-\sum_{r=0}^{k}(k-r+2)(k-r+ \\
& \text { 1) } \left.F[\mathrm{r}] F[\mathrm{k}-\mathrm{r}+2]-\frac{\phi_{4}}{\phi_{2}} R i\{\Theta[\mathrm{k}]+\xi \Phi[\mathrm{k}]\}\right]
\end{aligned}
$$

$\Theta[\mathrm{k}+2]=-\frac{P r}{\left(\frac{k_{n f}}{k_{f}}+N r\right)(k+1)(k+2)}[Q s * \Theta[\mathrm{k}]+$ $D u(k+1)(k+2) \Phi[\mathrm{k}+2]+\phi_{3} \sum_{r=0}^{k}(k-r+$ 1) $F[\mathrm{r}] \Theta[\mathrm{k}-\mathrm{r}+1]]$

$\Phi[\mathrm{k}+2]=\frac{s c}{(k+1)(k+2)}[\mathrm{Cr} \Phi[\mathrm{k}]-\mathrm{Sr} *$ $D u(k+1)(k+2) \Theta[\mathrm{k}+2]-\sum_{r=0}^{k}(k-r+$ 1) $F[\mathrm{r}] \Phi[\mathrm{k}-\mathrm{r}+1]]$ 
The boundary conditions (15) and (16) are also respectively transformed under DTM as:

$$
\begin{aligned}
& F[0]=S, F[1]=1, \Theta[0]=1, \Phi[0]=1 \\
& F[2]=a, \Theta[1]=b, \Phi[1]=c(\text { say })
\end{aligned}
$$

Where $F[\mathrm{k}], \Theta[\mathrm{k}], \Phi[\mathrm{k}]$ are differential transformed functions of $f(\eta), \theta(\eta), \phi^{*}(\eta)$ respectively and unknown quantities $a, b, c$ are constants to be determined from boundary condition (16). Now by using conditions (34) and (35) in Equations (31)(33), by means of the recursive method, we first calculate the values of $F[\mathrm{k}], \Theta[\mathrm{k}]$, and $\Phi[\mathrm{k}]$ for $k=0,1,2,3, \ldots$ and then substituting all values of $F[\mathrm{k}], \Theta[\mathrm{k}]$, and $\Phi[\mathrm{k}]$ thus obtained and using parametric values as $P r=6.2, \quad N r=0.8, \quad S c=1.2$, $D u=0.06, S r=0.8, Q s=0.4, C r=M=0.5, R i=0.7$, by means of equation (23) we have obtained finally the solutions of Equations (12), (13) and (14) in series expansion form as follows:

$$
\begin{aligned}
& f(\eta)=0.5+\eta+a * \eta^{2}+(0.1776192984- \\
& 0.1684638808 * a) * \eta^{3}+(0.1838356665 * a- \\
& 0.02458031928 * b-0.01229015964 * c- \\
& 0.02244182724) * \eta^{4}+(0.01331770137- \\
& 0.02649812180 * a+0.03369277616 * a^{2}+ \\
& 0.006547063287 * b+0.003035493151 * c) * \\
& \eta^{5}+ \\
& (0.001797159969 * a+0.0008696802066 * b- \\
& 0.00003522450468 * c-0.001526990420+ \\
& 0.03369277616 * a *(0.1776192984- \\
& \left.0.1684638808 * a)-0.002838007913 * a^{2}\right) * \\
& \eta^{6}+\cdots
\end{aligned}
$$

$$
\begin{aligned}
& \theta(\eta)=1+b * \eta+(-0.8568712054 * b- \\
& 0.8171003454) * \eta^{2}+(-0.3316397434 * b+ \\
& 0.4667665054-0.02248900034 * c) * \eta^{3}+ \\
& (0.7286503479 * b+0.3566810078+ \\
& 0.01373672587 * c-0.2856237353 * a * b) * \\
& \eta^{4}+(-0.05511326301 * b-0.3921771598+ \\
& 0.008761583137 * c+0.09789710174 * a * b- \\
& 0.3427484822 * a *(-0.8568712054 * b- \\
& 0.8171003454)-(0.1713742411 * \\
& (0.1776192984-0.1684638808 * a)) * b) * \\
& \eta^{5}+\cdots
\end{aligned}
$$

$$
\begin{aligned}
& \phi^{*}(\eta)=1+c * \eta+(0.8873646785 * b+ \\
& 1.084416331-0.3647683210 * c) * \eta^{2}+ \\
& (0.1087659456 * b-0.7470844729- \\
& 0.03288548832 * c) * \eta^{3}+(-0.9385002489 * \\
& b-0.4358093626+0.06254778388 * c+ \\
& 0.2957882261 * a * b-0.1215894403 * a * c) * \\
& \eta^{4}+(0.1752906863 * b+0.6085526301- \\
& 0.01184457690 * c-0.1445386354 * a * b+ \\
& 0.3549458714 * a *(-0.8568712054 * b- \\
& 0.8171003454)+(0.1774729357 * \\
& (0.1776192984-0.1684638808 * a)) * b+ \\
& 0.01774079039 * a * c-0.1459073284 * a * \\
& (0.8873646785 * b+1.084416331- \\
& 0.3647683210 * c)-(0.07295366419 * \\
& (.1776192984-0.1684638808 * a)) * c) * \eta^{5}+ \\
& \ldots
\end{aligned}
$$

The solutions (36) - (38) can be extended to a sufficient order of accuracy but these equations diverge around the large value of $\eta$. So the above solution forms do not converge and thus do not satisfy unbounded boundary conditions, i.e. expression (16). Therefore it is necessary to combine the series solution as obtained by DTM to the Padé approximant so to provide an effective tool to handle the unbounded boundary conditions. So we apply Padé approximation to Equations (36)-(38) such that the equations are represented in the form of Equation (25) and on using the unbounded boundary conditions (16), the values of unknown constants $a, b, c$ for above mentioned parametric values, are obtained as: $a=-0.271229, b=-1.3555, c=10.8829$,

where the respective Padé approximant solutions for Equations (36)-(38) are finally calculated up to $[8,8]$ order of magnitude as:

$$
\begin{aligned}
& f(\eta)_{[8,8]}= \\
& \left(1-4.473086586 * \eta+2.567627193 * \eta^{2}-\right. \\
& 3.750145059 * \eta^{3}+3.588743618 * \eta^{4}- \\
& 1.982437082 * \eta^{5}+0.8185558133 * \eta^{6}- \\
& \left.0.2782313998 * \eta^{7}+0.03664508821 * \eta^{8}\right) /(1- \\
& 4.360315586 * \eta+1.514555389 * \eta^{2}- \\
& 0.6291037399 * \eta^{3}+0.2902815463 * \eta^{4}- \\
& 0.003166304833 * \eta^{5}-0.02893727316 * \eta^{6}- \\
& \left.0.01255716760 * \eta^{7}-0.006758205048 * \eta^{8}\right)
\end{aligned}
$$


$\theta(\eta)_{[8,8]}=$

$\left(1+1.270069667 * \eta-1.631453050 * \eta^{2}+\right.$ $.6804423270 * \eta^{3}+1.858082044 * \eta^{4}+$ $52.31874421 * \eta^{5}+174.9547754 * \eta^{6}-$ $\left.62.68078239 * \eta^{7}+3.759759332 * \eta^{8}\right) /(1+$ $3.458379667 * \eta+4.878554274 * \eta^{2}+$ $3.222883171 * \eta^{3}-.5761089086 * \eta^{4}+$ $57.76389740 * \eta^{5}+295.5849305 * \eta^{6}+$ $\left.504.7512521 * \eta^{7}+556.5150646 * \eta^{8}\right)$

$\phi^{*}(\eta)_{[8,8]}=(1+9.221219160 * \eta-$

$24.37079196 * \eta^{2}-19.74681504 * \eta^{3}-$

$2.157896194 * \eta^{4}+.1108960795 * \eta^{5}-$

$.8219295342 * \eta^{6}+1.502854335 * \eta^{7}+$

$\left..4723352089 * \eta^{8}\right) /(1-1.661680833 * \eta-$

$2.198741959 * \eta^{2}-1.358909915 * \eta^{3}-$

$.4235662406 * \eta^{4}-0.001287466035 * \eta^{5}+$ $.1681334261 * \eta^{6}+0.07082330672 * \eta^{7}+$ $0.03633058228 * \eta^{8}$ )

\section{Numerical simulations and data analysis}

To validate the outcome of the study, numerical simulations of the results in terms of a set of chosen values of the governed physical parameters influencing the flow, are made. The base fluid is considered as water $\left(\mathrm{H}_{2} \mathrm{O}\right)$, whose Prandtl number is taken as 6.2 and the nanoparticles are chosen as Aluminum Oxide $\left(\mathrm{Al}_{2} \mathrm{O}_{3}\right)$, whose maximum volume fraction is considered as $5 \%$. In the investigation, the following parametric values are adopted for computations: $P r=6.2, N r=0.8, S c=1.2, D u=0.06$, $S r=0.8, Q s=0.4, C r=M=0.5, R i=0.7$. All graphs therefore will correspond to these values unless it is specifically mentioned.

Figures 1-3 portray the transverse magnetic field effect on the fluid velocity, temperature and concentration distributions respectively. It is seen that the velocity profile decreases with an increasing strength of the magnetic field effect, applied normaly to the fluid flow. The magnetic field produces a resistive force known as the Lorentz force, in the free convection region along the axial direction, which is opposing the flow. As this force acts as an obstruction to the flow, it subsequently extends the boundary layer thickness all over the flow regime. It can also be observed that the effect of the magnetic field is much stronger near to the surface of the plate. Again an additional amount of work needs to be done by the fluid particles to overcome from the obstacle imposed by the influence of the magnetic field. This additional work done also produces heat in the region and thus the temperature field increases with the increasing strength of the magnetic field effect, as shown in Figure 2. Also, it is noticed that the concentration profile decreases with the increasing values of the magnetic field parameter, as described in Figure 3. The rise in temperature fluxes probably increases the transport phenomenon of mass near the plate region as such the thickness of the concentration boundary layer diminishes. It is also observed from the figures that the effect of the magnetic field parameter is seem to be very minor on velocity profiles when compared to the temperature and concentration profiles.

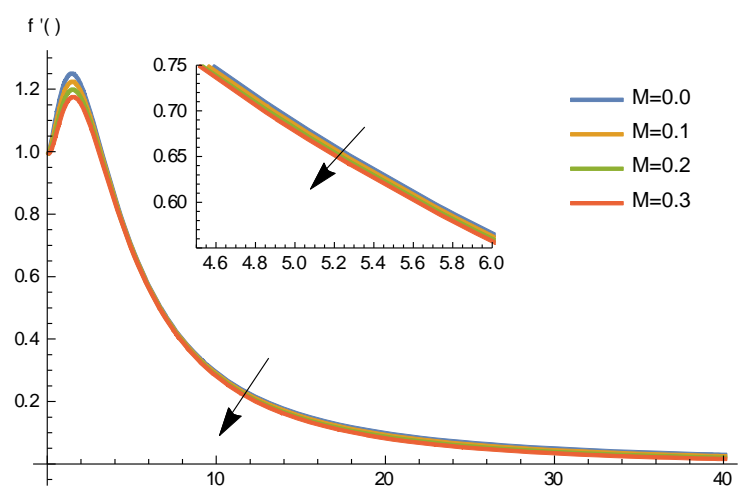

Fig. 1. Variation of velocity profiles for different values of magnetic parameter.

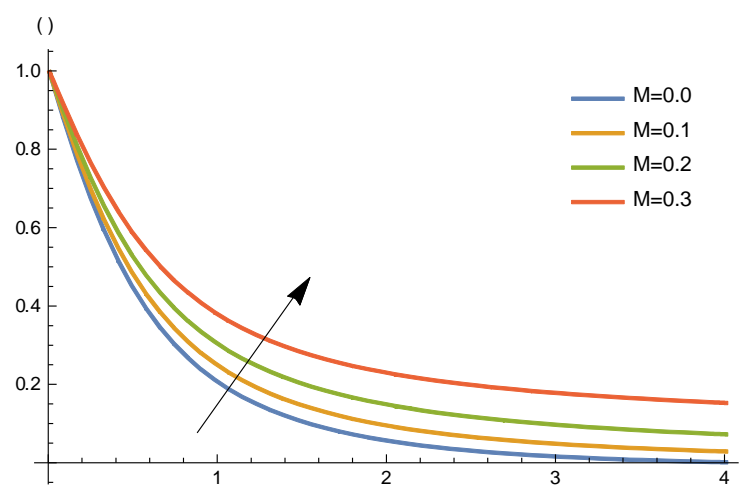

Fig. 2. Variation of temperature profiles for different values of magnetic parameter. 


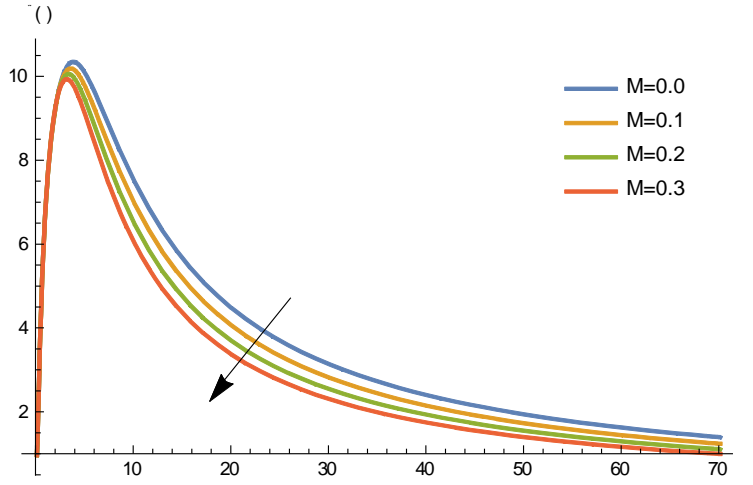

Fig. 3. Variation of concentration profiles for different values of magnetic parameter.

Figures 4-6 demonstrate the effect of Prandtl number on the fluid velocity, temperature and concentration profiles respectively. The Prandtl number is a dimensionless parameter which is defined to be the ratio of momentum diffusivity by thermal diffusivity. Thus an increase in this number corresponds to a higher momentum diffusivity as compared to thermal diffusivity. Therefore, an increase in the value of Prandtl number increases the momentum diffusivity and as a result, the internal friction of the nanofluid increases and consequently the momentum boundary layer thickness decreases. Also with an increase in the values of Prandtl number, as the temperature fluxes reduce, the thermal boundary layer thickness decreases and consequently the molar concentration of the constituents increases and this escalates the concentrations within the boundary layer. As a result, it is observed that, as Prandtl number increases, the velocity profile, as well as the temperature profile, decreases, whereas an inverse phenomenon is observed for the concentration profiles. From the figures, we observe that with an approximate $10.71 \%$ increase in the values of $\mathrm{Pr}$, the velocity and temperature profiles are respectively reduced by $8.9 \%$ and $13.88 \%$, whereas the concentration profiles are increased by $21.72 \%$.

The effect of thermal radiation on temperature and concentration profiles are revealed in Figure 7 and Figure 8 respectively. It is seen that an increment of thermal radiation parameter maximizes the temperature profiles and minimizes the concentration profiles. The presence of thermal radiation parameter in the flow domain helps in the process of thermal diffusion by increasing the temperature fluxes within the thermal boundary layer. This speed up the rise in temperature.

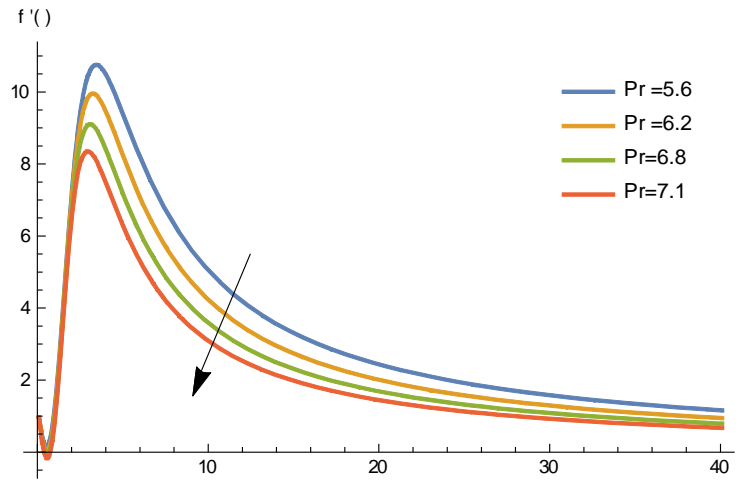

Fig. 4. Variation of velocity profiles for different values of Prandtl number.

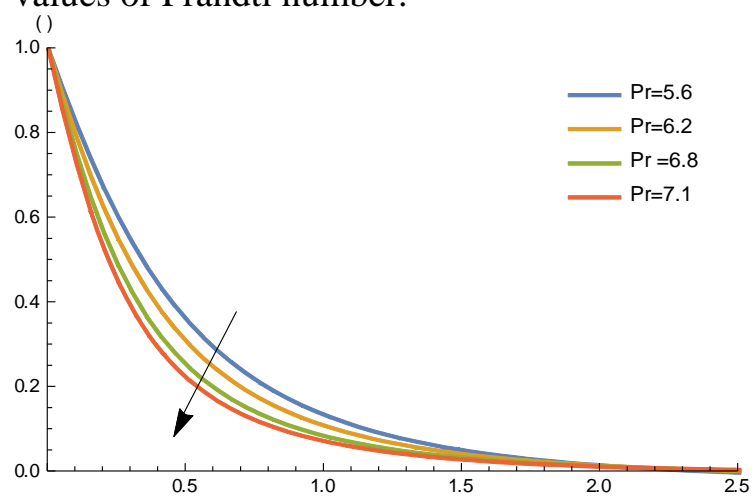

Fig. 5. Variation of temperature profiles for different values of Prandtl number.

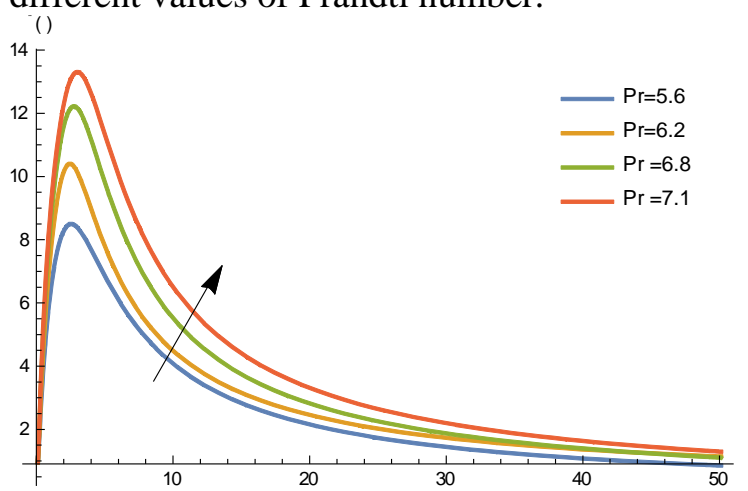

Fig. 6. Variation of concentration profiles for different values of Prandtl number.

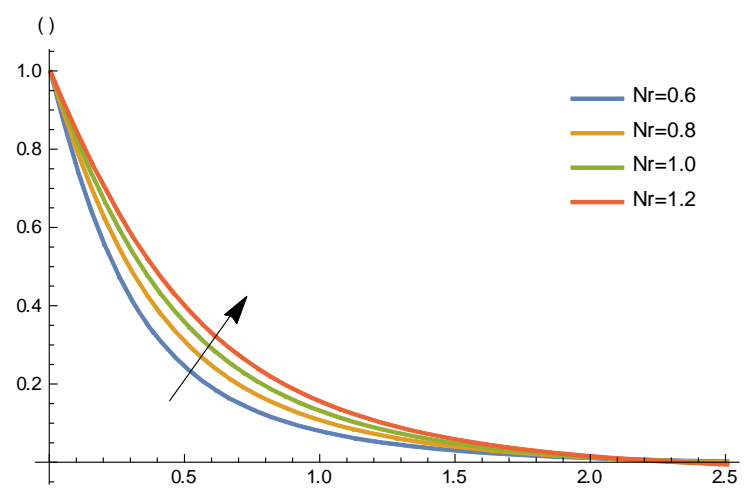

Fig. 7. Variation of temperature profiles for different values of thermal radiation parameter. 


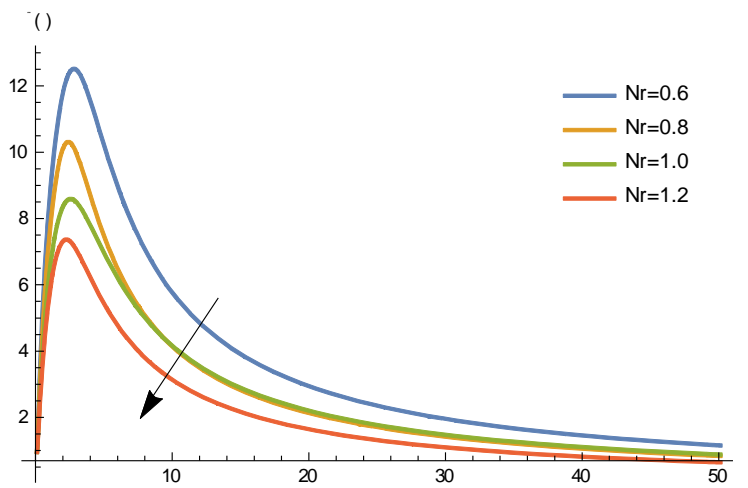

Fig. 8. Variation of concentration profiles for different values of thermal radiation parameter.

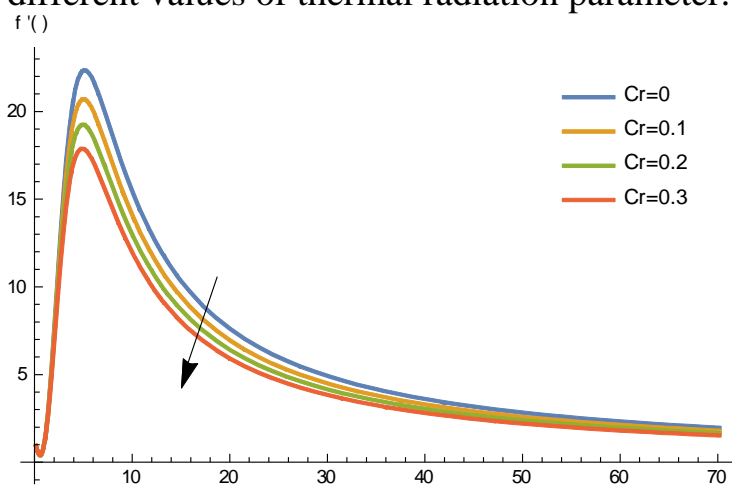

Fig. 9. Variation of velocity profiles for different values of chemical reaction parameter.

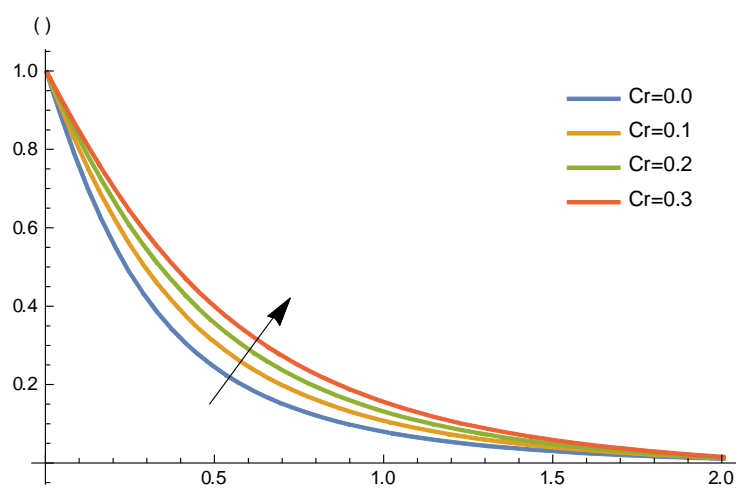

Fig. 10. Variation of temperature profiles for different values of chemical reaction parameter.

The rise in temperature of the fluid particles thinner the molar fluxes, which is why the concentration profiles are decreased. Also a $25 \%$ advancement in the values of $\mathrm{Nr}$, the temperature profiles advance by approximately $15.65 \%$ and the concentration profiles reduce by approximately $16.75 \%$.

Figures 9 - 11 are presented to study the effect of chemical reaction parameter on the fluid velocity, temperature, and concentration profiles respectively.With higher values of chemical reaction parameter, the transport of molar species from high concentration zone (plate region) to the low concentration zone (free-stream region) upsurges, which declines the growth of concentration boundary layer and thus lowered the concentrations near the plate region. Due to the dilution of concentrations near the plate region, the effect of mass buoyancy force minimizes. This tends to deceleration of the axial flow rate, thus diminishes the axial velocity. The first order chemical reaction between water (solvent) and the nanoparticles $\mathrm{Al}_{2} \mathrm{O}_{3}$ (solutes) is a thermodynamically favorable, homogeneous, and highly exothermic reaction with an enthalpy of reaction $280 \mathrm{kj} / \mathrm{mol} \mathrm{H}^{2}$ at $50-100^{\circ} \mathrm{C}$. Because of the generation of the large temperature gradient, the temperature profile shoots up. This rises the temperature of the fluid particles. It is observed from Figure 11 that, the effect of chemical reaction parameter is very prominent close to the plate surface and is not of much substantial outside the boundary layer. It is also found that, doubling the value $\mathrm{Cr}$, results in an increment of nearly $17 \%$ for the temperature profiles but a decrement of about $7 \%$ and $14 \%$ respectively for velocity and concentration profiles are reported.

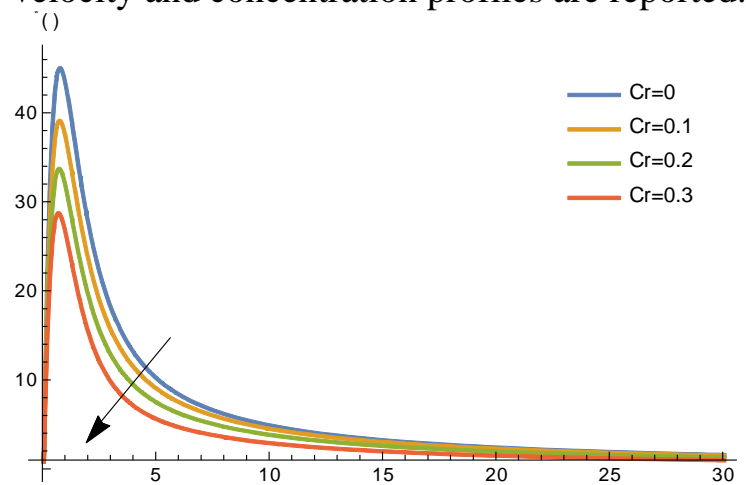

Fig. 11. Variation of concentration profiles for different values of chemical reaction parameter.

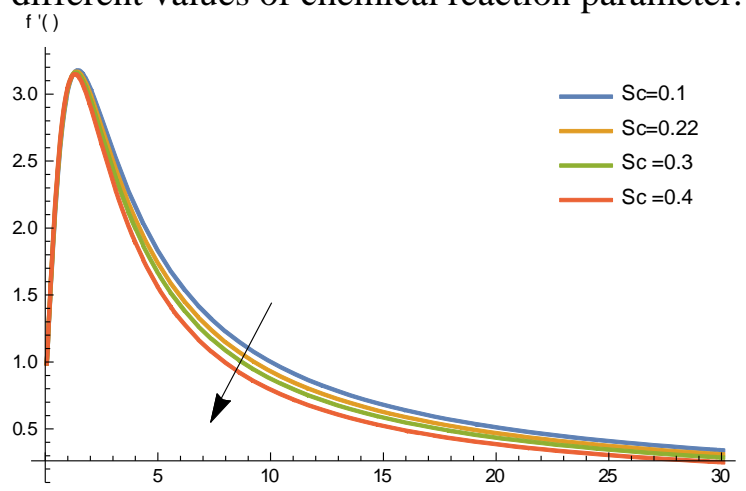

Fig. 12. Variation of velocity profiles for different values of Schmidt number.

The influence of Schmidt number on the velocity, temperature and concentration profiles are represented in Figures 12-14 respectively. It is well 
known to the literature that, Schmidt number is the ratio of momentum diffusivity to molar diffusivity and relates the relative thickness of the hydrodynamic boundary layer to the concentration boundary layer. In view of that, as the values of Schmidt number advances, the mass diffusivity minimizes and as a result the concentration profile of the nanofluid hinders. Due to the growth of the thickness of the velocity boundary layer, affected by the momentum diffusivity rate, the flow rate gets retarded, which results in slow down the motion with a reduction in values of axial velocity. On the other hand, due to the growth of the velocity boundary layer, the influence of frictional forces rises. This allows the constituents to do a more mechanical effort in the expense of heat energy. The heat energy that generates due to the momentum diffusion, automatically upsurge the temperature within the boundary layer. From calculation, we obtained that velocity and concentration profiles are reduced by approximately $0.41 \%$ and $26.57 \%$ respectively, whereas temperature profiles are increased by $3.67 \%$ when the values of Schmidt number is increased by $120 \%$.

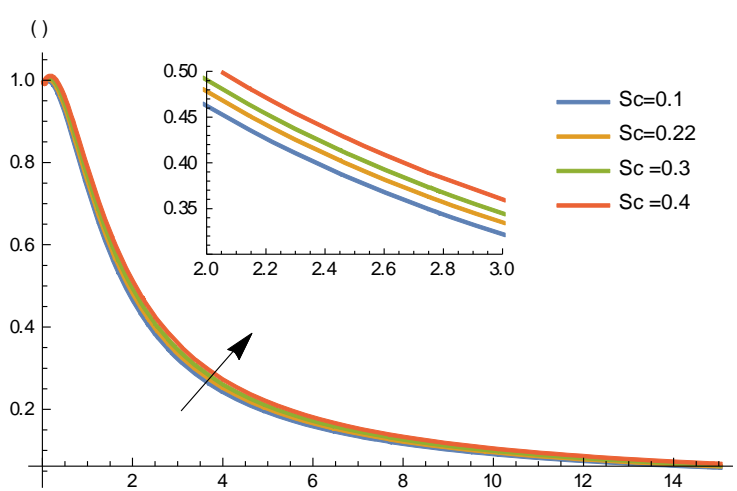

Fig. 13. Variation of temperature profiles for different values of Schmidt number.

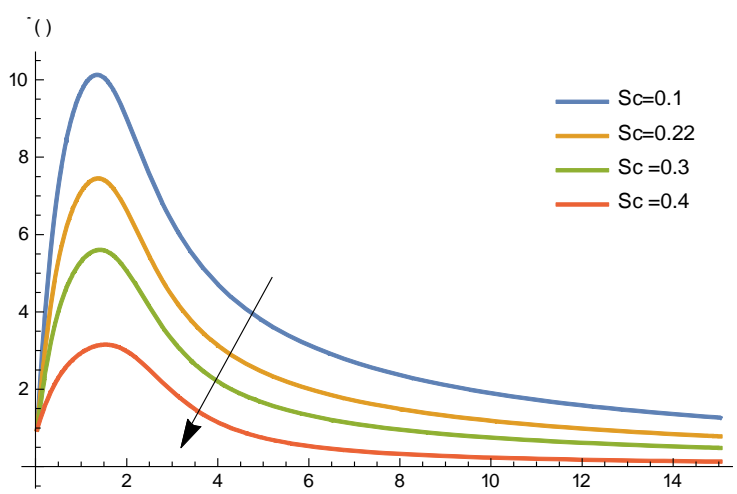

Fig. 14. Variation of concentration profiles for different values of Schmidt number.
Figures 15-17 aimed to shed light for heat source effect on the fluid velocity, temperature, and concentration profiles respectively. It is observed that, with increasing values of heat source parameter $(Q s>0)$, the velocity and temperature profiles get maximized, whereas a reverse effect is seen for the concentration profiles. This happens, because with increasing heat source parameter, an additional amount of heat fluxes generate, which is augmented with the heat fluxes and thereby expands the thickness of the thermal boundary layer. This effect is observed to proliferate the temperature profiles near the plate surface. On the other hand, due to the upsurge of temperature near the plate surface, the molar concentration of the fluid constituents gets reduced and as a result, the concentration of fluid particles falls gradually. Again the rise in temperature helps in rising the kinetic energy of the particles which also increases the thermal buoyancy force. This in turn accelerates the flow rate thereby increasing the axial fluid velocity. Numerical calculations have shown that the velocity and temperature profiles are increased by $10.65 \%$ and $14.06 \%$ respectively with $33 \%$ increament in the values of $Q s$, while reduces the concentration profiles by nearly $12.5 \%$.

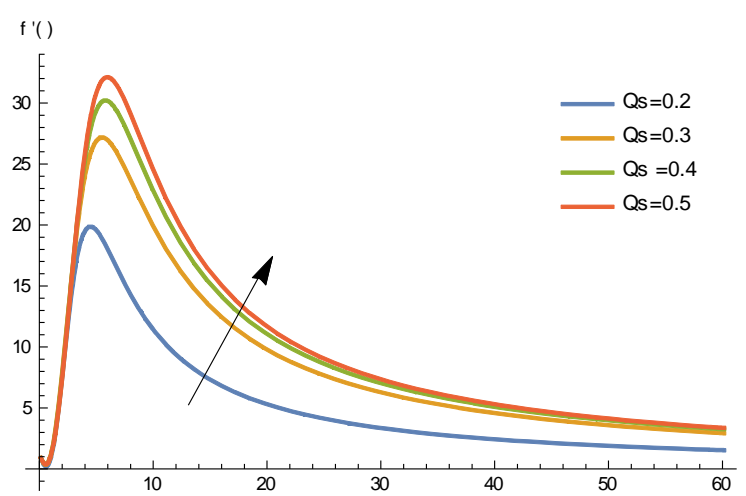

Fig. 15. Variation of velocity profiles for different values of heat source parameter.

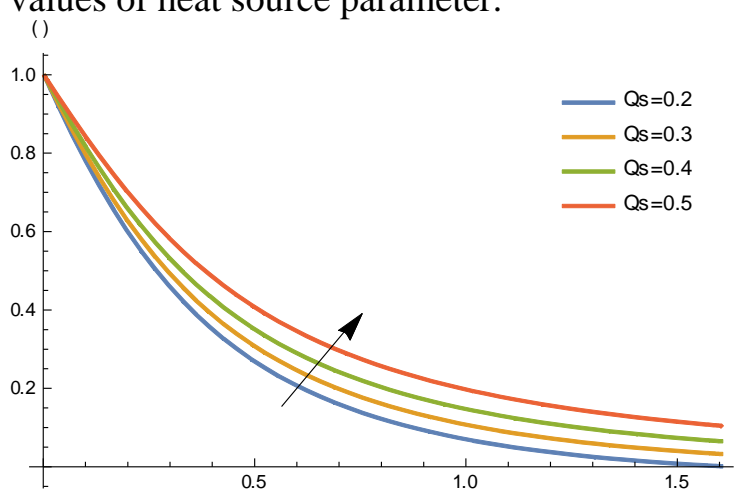

Fig. 16. Variation of temperature profiles for different values of heat source parameter. 


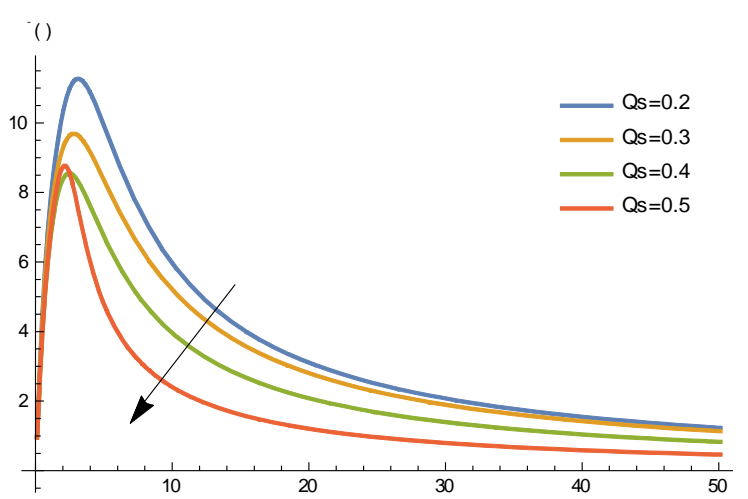

Fig. 17. Variation of concentration profiles for different values of heat source parameter.

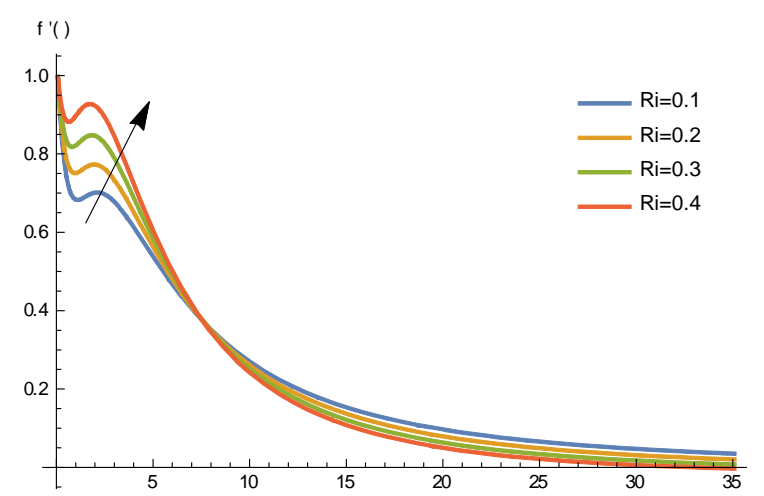

Fig. 18. Variation of velocity profiles for different values of Richardson number.

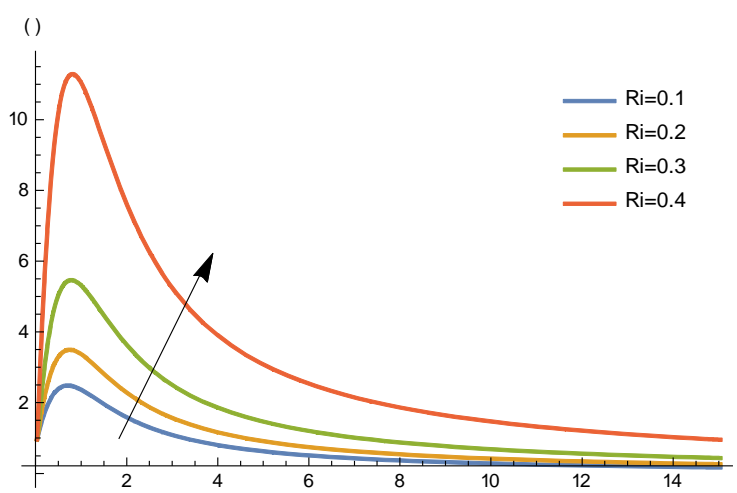

Fig. 19. Variation of temperature profiles for different values of Richardson number.

Figure 18 and Figure19 provide with the analysis on the variation of Richardson number over the fluid velocity and temperature profiles respectively. Since Richardson number physically signifies the ratio of buoyancy force term to the viscous term, thus an increase in the values of Richardson number, increases the buoyancy effects which in turn increases the kinetic energy. Again an increase in kinetic energy increases the flow rate and ultimately the velocity profiles get increased. On the other hand, an increase in Richardson number signifies the strength of the free convection phenomenon which reflects the strength of the free convection current near the plate surface, produced due to the considerable temperature difference of the fluid particles near the plate surface and the ambient temperature in the free stream region. This situation reflects physically as an increment in temperature profiles. Also, a rise in the velocity profile is significant close to the plate due to the effect of the produced buoyancy force. It is witnessed from the figures that, the effect of Richardson number is not much substantial on velocity profiles but is significant on temperature profiles and as such, when the value of $R i$ is increased by $50 \%$, the velocity profiles is increased by only $9.4 \%$ but the temperature profiles are found to be increased by a whooping $55.31 \%$ near the plate.

The Soret and Dufour effects are generally studied together, but we explore the influences of Soret and Dufour number separately on the velocity, temperature, and concentration distributions of the flow to clearly witness their individual effects. The effect of Soret number on velocity, temperature, and concentration distributions are respectively sketched in Figures 20-22. It is seen that, with increment in the values of Soret number, the velocity and concentration distributions increases whereas the exact opposite trend is observed for the temperature distribution of the flow. Since Soret number is defined as the effect of the ratio of temperature difference to the concentration difference, hence larger Soret number stands for a higher temperature difference and as a result with increasing values of Soret number the temperature distributions minimizes, consequently the concentration distributions maximizes. Fluid velocity also rises due to greater thermal diffusion. It is interesting to observe that, the effect of Soret number is marginal in the concentration profiles of the fluid flow. It is perceived from the figures that, Soret number has a microscopic but significant effect on every profile so that a $6.25 \%$ increase in the value of $S r$, velocity profiles are increased by $4.44 \%$, temperature profiles are decreased by $2.08 \%$, and the concentration profiles are increased by only $1.35 \%$. Figures 23 - 25 exhibit the influence of Dufour number on the velocity, temperature and concentration profiles of the flow respectively. Dufour number is described as the effect of the ratio of concentration difference to the temperature 
difference, which is exactly reversed to that of the Soret number. Hence with high values of Dufour number, the thermal and solutal buoyancy forces improve the convective velocity and consequently leads to increase the temperature of the fluid while retarding the concentration species. The Dufour number shows more effectiveness on the profiles as compared to Soret number. This can be verified form the fact that, when the Dufour number is increased by $22.2 \%$, both velocity and temperature profiles are increased by nearly $10 \%$, also the cpncentration profiles are decreased by approximately $11 \%$.

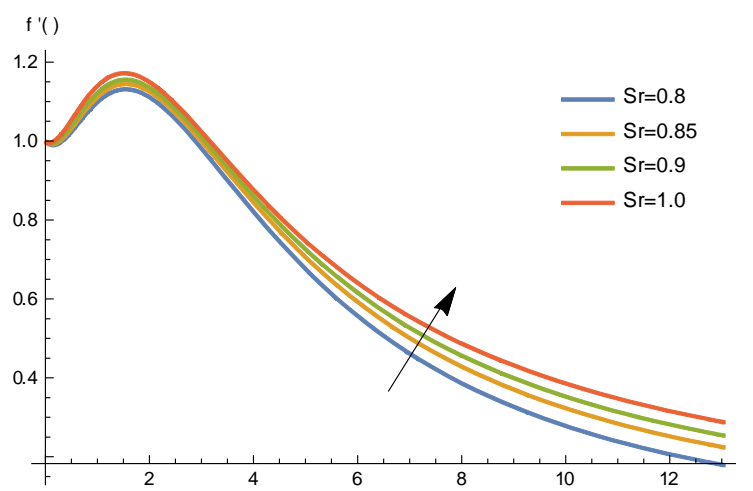

Fig. 20. Variation of velocity profiles for different values of Soret number.

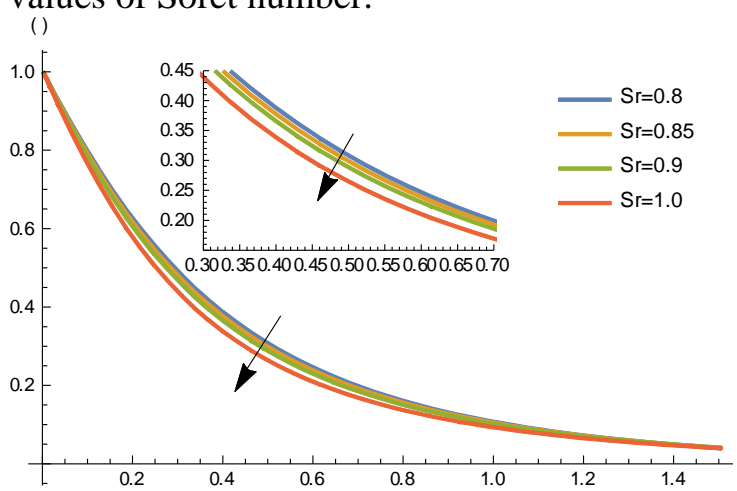

Fig. 21. Variation of velocity profiles for different values of Soret number.

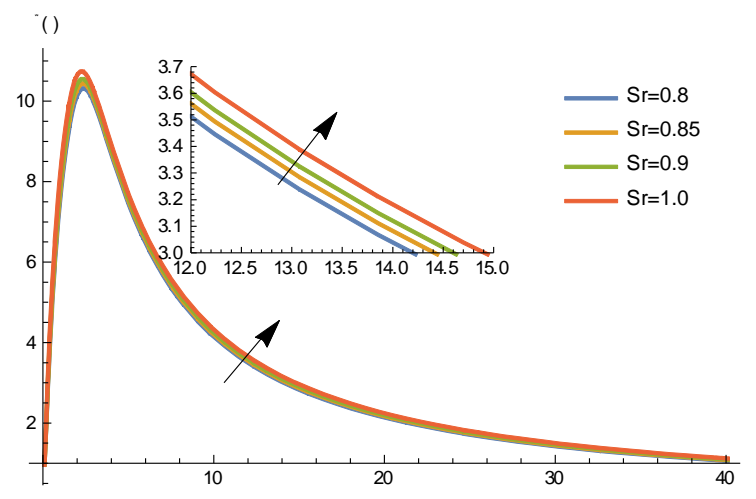

Fig. 22. Variation of concentration profiles for different values of Soret number.

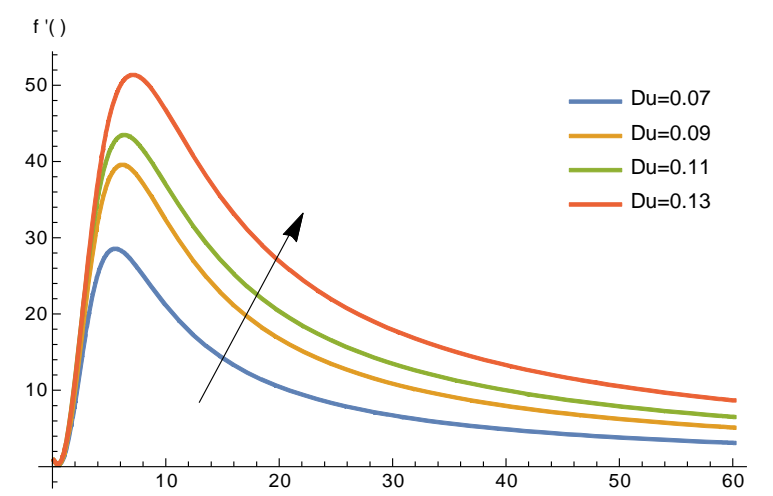

Fig. 23. Variation of velocity profiles for different values of Dufour number.

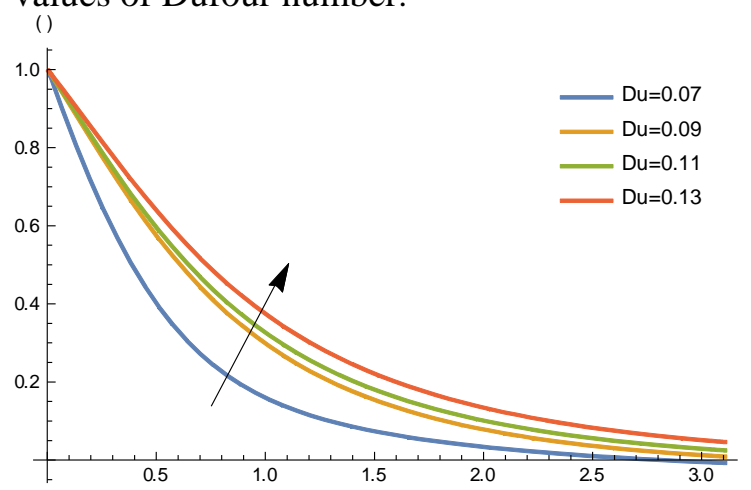

Fig. 24. Variation of temperature profiles for different values of Dufour number.

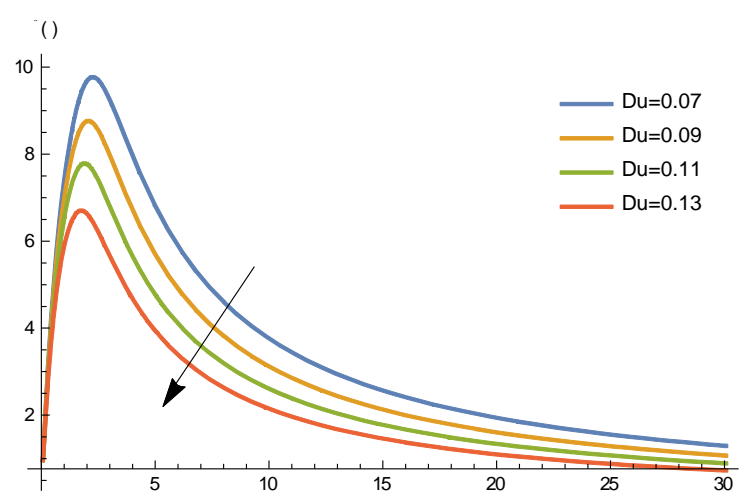

Fig. 25. Variation of concentration profiles for different values of Dufour number.

Table 1 is prepared to visualize the effects of nanoparticle volume fraction, thermal radiation and porous medium parameter on fluid velocity distributions and skin friction coefficient. It is interesting to observe that, as the volume fraction of nanoparticles increases, the flow velocity reduces marginally, this is because of the fact that with a higher number of nanoparticles in the base fluid, it becomes denser and as a result internal friction of 
the fluid increases which also increases the skin friction co-efficient. Also increment in values of thermal radiation parameter results in the increment of the velocity distributions, while reduces the skinfriction co-efficient significantly. Similar results are seen with the presence of $k_{p}$.

Table 1. Variation of velocity profiles and skin friction coefficient for different parameters.

\begin{tabular}{|c|c|c|c|c|}
\hline$\phi$ & $N_{R}$ & $k_{p}$ & $f^{\prime}(0.5)$ & $\tau_{w}$ \\
\hline 0.02 & 0.8 & 0.5 & 0.84163703 & 0.98861581 \\
\hline 0.03 & 0.8 & 0.5 & 0.83665338 & 1.06240453 \\
\hline 0.04 & 0.8 & 0.5 & 0.83055773 & 1.14398117 \\
\hline 0.05 & 0.6 & 0.5 & 0.71559999 & 1.93460524 \\
\hline 0.05 & 1.0 & 0.5 & 0.82993881 & 1.37754618 \\
\hline 0.05 & 1.2 & 0.5 & 0.96214985 & 0.64842741 \\
\hline 0.05 & 0.8 & 0.8 & 0.56456233 & 4.62293909 \\
\hline 0.05 & 0.8 & 1.0 & 0.58537102 & 4.55513926 \\
\hline 0.05 & 0.8 & 1.2 & 0.60318902 & 4.50530115 \\
\hline
\end{tabular}

Table 2 presents the parametric effects of $\mathrm{Nr}, \mathrm{Ri}, \mathrm{M}, \mathrm{Cr}, \emptyset$ on the Nusselt number and Sherwood number respectively. As seen from the table, with increasing values of thermal radiation parameter and Richardson's number, Nusselt number decreases and Sherwood number increases. As the thermal diffusivity increases due to increase in temperature fluxes affected by an increase in thermal radiation parameter, the transport of heat energy from the plate surface to the ambient region is thus declines. This is why, the rate of heat transfer (Nusselt number) gets reduce. On the other hand, a raise in temperature at the plate surface encourages the migration of species, which saw in increasing the mass transfer rate (Sherwood number). An enlarge in values of Richardson's number is seen to boost the thermal buoyancy force, which is responsible to expand the thermal boundary layer thickness, thereby reducing the thermal transport rate, while improving the mass transfer rates. Again, due to increase in the magnetic field parameter, the thermal diffusivity increases, which restricts the thermal energy transport, while encouraging the cross flow motion of constituents, results of which the mass transfer rate gets accelerate. The amplification of first order chemical reaction parameter in the flow domain is to decrease both the thermal and solutal diffusivity co-efficient, which allow the thermal and mass transport rates to step up steadily. On the other hand, an increase in volume fraction of nanoparticles is clearly observed to increase the transport rates. As the volume fractions of the nanoparticles increases, the number of solid to - solid interaction is also increases. This helps to migrate the heat energy from one surface to another along the cross flow direction and thus increase the heat transfer rate. On the other hand, a large gathering of nanoparticles near the surface prompted to transport masses from higher concentration zone to lower concentration zone in the cross flow direction, which is seen to increase the mass transfer rate.

Table 2. Variation of the Nusselt number and Sherwood number for different parameters.

\begin{tabular}{|l|l|l|l|l|l|l|}
\hline$N_{R}$ & $R i$ & $M$ & $C_{r}$ & $\emptyset$ & $N u$ & $S h$ \\
\hline 0.6 & 0.7 & 0.5 & 0.5 & 0.05 & 4.386256 & 4.044416 \\
\hline 1.0 & & & & & 4.005083 & 4.447116 \\
\hline 1.2 & & & & & 3.262280 & 4.909534 \\
\hline 0.8 & 0.2 & & & & 7.044935 & 1.596341 \\
\hline & 0.3 & & & & 2.833955 & 3.319642 \\
\hline & 0.4 & & & & 2.487477 & 3.523830 \\
\hline & & 0.1 & & & 29.72926 & 16.18999 \\
\hline & & 0.2 & & & 28.56913 & 15.14759 \\
\hline & & 0.3 & & & 27.26422 & 14.08122 \\
\hline & & & 0.1 & & 6.189272 & 14.18271 \\
\hline & & & 0.2 & & 6.364905 & 14.24904 \\
\hline & & & 0.3 & & 6.792817 & 14.81931 \\
\hline & & & & 0.02 & 6.470283 & 16.64363 \\
\hline & & & & 0.03 & 6.957036 & 16.67587 \\
\hline & & & & 0.04 & 7.527147 & 16.80064 \\
\hline
\end{tabular}

Table 3. Nomenclature with corresponding measuring unit

\begin{tabular}{|l|l|}
\hline$A_{R}$ & $\begin{array}{l}\text { coefficient of Rosseland mean } \\
\text { spectral absorption }\left[\mathrm{m}^{-1}\right]\end{array}$ \\
\hline$b$ & stretching sheet parameter \\
\hline$B_{0}$ & magnetic field strength, $[\mathrm{T}]$ \\
\hline$C$ & concentration of nanofluid $\left[\mathrm{mol} / \mathrm{m}^{3}\right]$ \\
\hline$C_{f}$ & Skin friction coefficient \\
\hline$C_{M}$ & $\begin{array}{l}\text { mean fluid concentration at stretching } \\
\text { sheet }\left[\mathrm{mol} / \mathrm{m}^{3}\right]\end{array}$ \\
\hline$C_{p}$ & $\begin{array}{l}\text { specific heat at constant } \\
\text { pressure } \mathrm{J} / \mathrm{Kg} \cdot \mathrm{K}]\end{array}$ \\
\hline$C_{S}$ & $\begin{array}{l}\text { concentration } \\
{\left[4 \pi \mathrm{Cm}^{3} / \mathrm{mol}\right]}\end{array}$ \\
\hline$C_{\infty}$ & $\begin{array}{l}\text { freestream fluid concentration } \\
{\left[\mathrm{mol} / \mathrm{m}^{3}\right]}\end{array}$ \\
\hline
\end{tabular}




\begin{tabular}{|c|c|}
\hline $\mathrm{Cr}$ & chemical reaction parameter \\
\hline$D_{M}$ & species diffusivity $\left[\mathrm{m}^{2} / \mathrm{s}\right]$ \\
\hline$D u$ & Dufour number \\
\hline$E_{b}$ & black body emissive power $\left[\mathrm{W} / \mathrm{m}^{2}\right]$ \\
\hline$g$ & gravitational acceleration $\left[\mathrm{m} / \mathrm{s}^{2}\right]$ \\
\hline $\bar{K}$ & permeability parameter \\
\hline$k$ & thermal conductivity $[W / m \cdot K]$ \\
\hline$k_{l}$ & $\begin{array}{l}\text { first order dimensional chemical } \\
\text { reaction parameter }\end{array}$ \\
\hline$k_{p}$ & porous medium parameter \\
\hline$K_{T}$ & thermal diffusivity ratio $\left[\mathrm{m}^{2} / \mathrm{s}\right]$ \\
\hline$M$ & magnetic field parameter \\
\hline$N r$ & thermal radiation parameter \\
\hline $\mathrm{Nu}$ & Nusselt number \\
\hline $\mathrm{Pr}$ & Prandtl number \\
\hline$Q$ & dimensional heat source parameter \\
\hline$q_{r}$ & radiation heat flux $\left[\mathrm{Wm}^{-2}\right]$ \\
\hline Qs & heat source parameter \\
\hline$R i$ & Richardson number \\
\hline$S$ & mass flux parameter \\
\hline Sc & Schmidt number \\
\hline$S h$ & Sherwood number \\
\hline $\mathrm{Sr}$ & Soret number \\
\hline$T$ & temperature of nanofluid $[K]$ \\
\hline$T_{M}$ & $\begin{array}{l}\text { mean fluid temperature at stretching } \\
\text { sheet }[K]\end{array}$ \\
\hline$T_{\infty}$ & ambient temperature $[K]$ \\
\hline$u$ & $\begin{array}{l}\text { velocity } \\
{[\mathrm{m} / \mathrm{s}]}\end{array}$ \\
\hline$v$ & $\begin{array}{l}\text { velocity component along } y \text { direction } \\
{[\mathrm{m} / \mathrm{s}]}\end{array}$ \\
\hline$v_{M}$ & wall mass flux $[\mathrm{m} / \mathrm{s}]$ \\
\hline$\xi$ & concentration buoyancy parameter \\
\hline$\mu$ & dynamic viscosity $\left[\mathrm{Ns} / \mathrm{m}^{2}\right]$ \\
\hline$\rho$ & effective density $\left[\mathrm{Kg} / \mathrm{m}^{3}\right]$ \\
\hline$\sigma$ & electrical conductivity $[\mathrm{S} / \mathrm{m}]$ \\
\hline$\rho c_{p}$ & heat capacitance $\left[J / K \cdot m^{-3}\right]$ \\
\hline$v$ & $\begin{array}{l}\text { kinematic coefficient of viscosity } \\
{\left[\mathrm{m}^{2} / \mathrm{s}\right]}\end{array}$ \\
\hline$\rho \beta_{C}$ & solutal buoyancy parameter \\
\hline$\sigma_{S B}^{*}$ & $\begin{array}{l}\text { Stefan-Boltzmann constant } \\
{\left[\left(=5.6697 * 10^{-8}\right), W / m^{2} K^{4}\right]}\end{array}$ \\
\hline$\psi$ & stream function \\
\hline$\rho \beta_{T}$ & hermal buoyancy parameter \\
\hline$\alpha$ & thermal diffusivity $\left[\mathrm{m}^{2} / \mathrm{s}\right]$ \\
\hline
\end{tabular}

\begin{tabular}{|l|l|}
\hline$\beta$ & thermal expansion coefficient $\left[K^{-1}\right]$ \\
\hline$\emptyset$ & $\begin{array}{l}\text { volume fraction of solid particles in } \\
\text { the fluid [\%] }\end{array}$ \\
\hline$f$ & base fluid \\
\hline$n f$ & nanofluid \\
\hline$s$ & suspended solid nanoparticles \\
\hline
\end{tabular}

\section{Concluding remarks}

In the present study, we have analyzed heat and mass transfer phenomena of electrically conducting nanofluid over a linearly stretched vertical sheet under the influence of magnetic field effect, first order chemical reaction, thermal radiation, and heat source effect. The complementary effects, like Soret and Dufour effects, are also considered for the study. The odorless crystalline powder Aluminum Oxide $\left(\mathrm{Al}_{2} \mathrm{O}_{3}\right)$, which shows better single phase nanofluid model and can unearth many complex as well as interesting facts, is taken as the nanoparticle and the water is considered as the base fluid for the present investigation. We have outlined stimulating insights of the influence of different parameters on the concerned fluid properties by means of graphs and tables. Numerical simulations are made for different parametric values of magnetic field parameter $(M)$, Prandtl number $(P r)$, thermal radiation parameter $(\mathrm{Nr})$, chemical reaction parameter $(\mathrm{Cr})$, Schmidt number $(\mathrm{Sc})$, heat source parameter $(Q s)$, Richardson number $(R i)$, Soret number $(S r)$, Dufour number $(D u)$ and their effects on heat and mass transfer characteristics are investigated graphically in detail. Effect of volume fraction $(\emptyset)$, porous medium parameter $\left(k_{p}\right)$, and other significant parameters on skin-friction coefficient, Nusselt number $(\mathrm{Nu})$, and Sherwood number $(S h)$ are also studied. Based on the theoretical investigation, we can conclude that, velocity and concentration profiles are decreased with increasing values of $M$ whereas the temperature profiles get increased. Both velocity and temperature profiles are maximized with increasing values of $R i$, and the effect of $R i$ on temperature profiles is very much significant, as an increase of $50 \%$ in the values of $R i$, the temperature profiles are found to be increased by $55 \%$, near the plate. Temperature and velocity profiles are reduced with higher values of $P r$. Temperature profiles are enlarged with advancing values of $\mathrm{Nr}$ and concentration profiles are affected reversely. Higher values of $\mathrm{Cr}$ reduce concentration and velocity 
profiles but advance temperature profiles. Effect of $S c$ on concentration and temperature profiles is observed as very less significant. Concentration and velocity profiles are decreased by $0.41 \%$ and $26.57 \%$ respectively, whereas the temperature profiles are increased by $3.67 \%$, with $120 \%$ increase in the values of $S c$. With increasing values of $Q s$, both temperature and velocity profiles upsurge. Velocity profiles escalate for higher values of both $\mathrm{Sr}$ and $\mathrm{Du}$. But for larger values of $\mathrm{Sr}(\mathrm{Du})$ temperature profiles get decreased (increased), whereas concentration profiles are found to be increased (decreased). Skin-friction coefficient increases with increasing $\emptyset$ but decreases with $k_{p}$. Nusselt number increases with increasing values of $\emptyset$ and $\mathrm{Cr}$, whereas decreases with increasing values of $N r, R i, M$. Sherwood number increases with increasing values of $\emptyset, \mathrm{Nr}, \mathrm{Ri}$, and $\mathrm{Cr}$, whereas decreases with increasing values of $M$.

\section{Acknowledgement:}

D. Saha gratefully acknowledges the support of Department of Science and Technology (DST), Govt. of India, for providing DST-INSPIRE Fellowship no. DST/INSPIRE Fellowship/2015/IF150831

References:

[1] Choi, S.U.S., Enhancing thermal conductivity of fluids with nanoparticles, ASME International Mechanical Engineering Congress and Exposition, ASME Fluids Eng. Div., 231/MD 66, San Francisco, USA, 1995, pp. 99-105.

[2] Keblinski, P., Phillpot, S.R., Choi, S.U.S. and Eastman, J.A., Mechanisms of heat flow in suspensions of nano-sized particles (nanofluids), International Journal of Heat and Mass Transfer, Vol.45, No. 4, 2002, pp. 855863.

https://doi.org/10.1016/S00179310(01)00175-2

[3] Buongiorno, J., Convective transport in nanofluids, Journal of Heat Transfer, Vol. 128, No. 3, 2006, pp. 240-250. http://dx.doi.org/10.1115/1.2150834

[4] Sengupta, S. and Karmakar, A., Heat and mass transfer analysis on MHD mixed convection flow of radiative chemically heat generating fluid with viscous dissipation and thermo-diffusion effect, Frontiers in Heat and Mass Transfer(FHMT), 2018, $11-30$. http://dx.doi.org/10.5098/hmt.11.30

[5] El-Kabeir, S.M.M., Soret and Dufour effects on heat and mass transfer due to a stretching cylinder saturated porous medium with chemically-reactive species, Latin American Applied Research, Vol. 41, 2011,pp. 331337.

[6] Haile, E. and Shankar, B., Heat and mass transfer in the boundary layer of unsteady viscous nanofluid along a vertical stretching sheet, Journal of Computational Engineering, 2014, Article ID-345153. http://dx.doi.org/10.1155/2014/345153

[7] Sengupta, S., Heat and Mass Transfer Phenomena in MHD Flow: Modelling with Applications, Scientific Research Publishing, USA, 2014.

[8] Sheikholeslami, M., Hayat, T. and Alsaedi, A., MHD free convection of $\mathrm{Al} 2 \mathrm{O} 3$-water nanofluid considering thermal radiation: A numerical study, International Journal of Heat and Mass Transfer, Vol. 96, 2016, pp. 513524.

https://doi.org/10.1016/j.ijheatmasstransfer.20 16.01.059

[9] Hayat, T., Iqbal, R., Tanveer, A. and Alsaedi, A., Soret and Dufour effects in MHD peristalsis of pseudoplastic nanofluid with chemical reaction, Journal of Molecular Liquids, Vol. 220, 2016, pp. 693-706. https://doi.org/10.1016/j.molliq.2016.04.123

[10] Raju, R.S., Reddy, G.J., Rao, J.A. andRashidi, M.M., Thermal diffusion and diffusion thermo effects on an unsteady heat and mass transfer magneto-hydrodynamic natural convection Couette flow using FEM, Journal of Computational Design and Engineering, Vol. 3, No. 4, 2016,pp. 349-362. https://doi.org/10.1016/j.jcde.2016.06.003

[11] Yirga, Y. and Tesfay, D., Heat and mass transfer in MHD flow of nanofluids through a porous media due to a permeable stretching sheet with viscous dissipation and chemical reaction effects, International Journal of Mechanical, Aerospace, Industrial, Mechatronic and Manufacturing Engineering, Vol. 9, No. 5, 2015, pp. 709-716.

[12] Sheikholeslami, M., Ganji, D.D., Javed, M.Y. and Ellahi, M., Effect of thermal radiation on 
magnetohydrodynamics nanofluid flow and heat transfer by means of two phase model, Journal of Magnetism and Magnetic Materials, Vol. 374, 2015,pp. 36-43. https://doi.org/10.1016/j.jmmm.2014.08.021

[13] Reddy, P.S. and Chamkha, A.J., Soret and Dufour effects on MHD convective flow of Al2O3-water and $\mathrm{TiO} 2-$ water nanofluids past a stretching sheet in porous media with heat generation/absorption, Advanced Powder Technology, Vol. 27, 2016, pp. 1207-1218. https://doi.org/10.1016/j.apt.2016.04.005

[14] Rashidi, M.M., Ganesh, N.V., AbdulHakeem, A.K. and Ganga, B., Buoyancy effect on MHD flow of nanofluid over a stretching sheet in the presence of thermal radiation, Journal of Molecular Liquids, Vol. 198, 2014, pp. 234-238. https://doi.org/10.1016/j.molliq.2014.06.037

[15] Hayat, T., Muhammad, T., Shehzad, S.A. and Alsaedi, A., Similarity solution of three dimensional boundary layer flow of second grade nanofluid past a stretching surface with thermal radiation and heat source/sink, AIP Advances, Vol. 5, No. 1, 2015, Article ID 017107. https://doi.org/10.1063/1.4905780

[16] Bhatti, M.M. and Rashidi, M.M., Effects of thermo-diffusion and thermal radiation on Williamson nanofluid over a porous shrinking/stretching sheet, Journal of Molecular Liquids, Vol. 221, 2016, pp. 567573.

https://doi.org/10.1016/j.molliq.2016.05.049

[17] Saha, D. and Sengupta, S., Heat transfer analysis on $\mathrm{TiO} 2$-water nanofluid past a stretching sheet in magnetic field domain with heat generation, Proceedings of International Conference on Systems and Processes in Physics, Chemistry and Biology, India, pp. 21-24, (2018).

[18] Hayat, T., Imtiaz, M., Alsaedi, A. and Kutbi, M.A., MHD three-dimensional flow of nanofluid with velocity slip and nonlinear thermal radiation, Journal of Magnetism and Magnetic Materials, Vol. 396, 2015, pp. 3137.

https://doi.org/10.1016/j.jmmm.2015.07.091

[19] Karthikeyan, S., Bhuvaneswari, M., Sivasankaran, S. and Rajan, S., Soret and Dufour effects on MHD mixed convection heat and mass transfer of a stagnation point flow towards a vertical plate in a porous medium with chemical reaction, radiation and heat generation, Journal of Applied Fluid Mechanics, Vol. 9, No. 3, 2016, pp. 14471455.

http://eprints.um.edu.my/id/eprint/17881

[20] Sengupta, S., Free convective chemically absorption fluid past an impulsively accelerated plate with thermal radiation variable wall temperature and concentrations, Applications and Applied Mathematics, Vol. 10, No. 1, 2015, pp. 328-348.

[21] Ahmed, N., Sengupta, S. and Datta, D., An exact analysis for MHD free convection mass transfer flow past an oscillating plate embedded in a porous medium with Soret effect,Chemical Engineering Communications, Vol. 200, No. 4, 2012, pp. 494-513.

https://doi.org/10.1080/00986445.2012.70947 4

[22] Pal, D., Mandal, G. and Vajravalu, K. Soret and Dufour effects on MHD convectiveradiative heat and mass transfer of nanofluids over a vertical non-linear stretching/shrinking sheet, Applied Mathematics and Computation, Vol. 287-288, 2016, pp. 184-200. https://doi.org/10.1016/j.amc.2016.04.037

[23] Sengupta, S. and Ahmed, N., MHD free convective mass transfer flow of radiative uniform heat generation (absorption) fluid through a wavy permeable channel in the presence of Soret and Dufour effects,Canadian Journal of Physics, Vol. 95, No. 1, 2017, pp. 44-58. https://doi.org/10.1139/cjp-2014-0599

[24] Zhou, J.K., Differential transformation and its applications for electrical circuits, Huazhong Univ. Press, China, pp. 1279-1289, 1986.

[25] Hayat, T., Muhammad, T., Alsaedi, A. and Alhuthali, M.S., Magnetohydrodynamic threedimensional flow of viscoelastic nanofluid in the presence of nonlinear thermal radiation, Journal of Magnetism and Magnetic Materials, Vol. 385, 2015, pp. 222-229. https://doi.org/10.1016/j.jmmm.2015.02.046

[26] Zhang, C., Zheng, L., Zhang, X. and Chen, G., MHD flow and radiation heat transfer of nanofluids in porous media with variable surface heat flux and chemical reaction, Applied Mathematical Modelling, Vol. 39, No. 1, 2015, pp. 165-181. 
https://doi.org/10.1016/j.apm.2014.05.023

[27] Sheikholeslami, M. and Ganji, D.D., Nanofluid flow and heat transfer between parallel plates considering Brownian motion using DTM,Computer Methods in Applied Mechanics and Engineering, Vol. 283, 2015, pp. 651-663.

https://doi.org/10.1016/j.cma.2014.09.038

[28] Satya-Narayana, P.V., Venkateswarlu, B. and Venkataramana, S., Thermal Radiation and Heat Source Effects on a MHD Nanofluid Past a Vertical Plate in a Rotating System with Porous Medium, Heat Transfer-Asian Research, Vol. 44, No. 1, 2015, pp. 1-19. https://doi.org/10.1002/htj.21101

[29] Magyari, E. and Pantokratoras, A., Note on the effect of thermal radiation in the linearized Rosseland approximation on the heat transfer characteristics of various boundary layer flows, International Communications in Heat and Mass Transfer, Vol. 38, No. 5, 2011, 554556.https://doi.org/10.1016/j.icheatmasstransf er.2011.03.006 